\title{
Evaluation of interface quality in organ-cultured lamellar corneal transplants
}

This article was published in the following Dove Press journal:

Clinical Ophthalmology

5 July 2012

Number of times this article has been viewed

\author{
Armin Wolf',* \\ Burkhard von Jagow ${ }^{2, *}$ \\ Daniel Kook' \\ Elisabeth M Messmer' \\ Carlo A Lackerbauer' \\ Anselm Kampik' \\ Thomas Kohnen ${ }^{2}$ \\ Martin Grueterich' \\ 'Department of Ophthalmology, \\ Ludwig-Maximilians-Universität, \\ München, ${ }^{2}$ Department of \\ Ophthalmology, Johann Wolfgang \\ Goethe-Universität, Frankfurt am \\ Main, Germany \\ *These authors have contributed \\ equally
}

\begin{abstract}
Background: With increasing numbers of lamellar keratoplasties, eye banks are challenged to deliver precut lamellar donor tissue. In Europe, the most common technique of corneal storage is organ culture which requires a deswelling process before surgical processing. The aim of this study was to investigate the influence of different deswelling times on the cutting plane quality after microkeratome-assisted lamellar dissection.
\end{abstract}

Methods: Eight paired donor corneas (16 specimens) not suitable for transplantation were organ cultured under standard conditions at the Eye Bank of the Ludwig-Maximilians Universität, Munich, Germany. Pairs of corneal buttons were analyzed during the deswelling process in dextrane-containing medium. While one cornea was cut at an early time point during the deswelling process and put back into deswelling medium thereafter, the partner cornea was completely deswollen and dissected after 72 hours. Specimens were then further processed for scanning electron microscopy. Surface quality was assessed both digitally using Scanning Probe Imaging Processing software, and manually by three blinded graders.

Results: The corneal buttons processed at the beginning of the deswelling process had a smoother surface when compared to the partner cornea that was cut at the end of the deswelling process. In our setting, no relevant difference was detectable between manual and automated microkeratome dissection.

Conclusion: For lamellar keratoplasty, organ-cultured corneas should be processed at an early stage during the deswelling process. We interpret the smoother dissection plane during early deswelling as a result of mechanical properties in a highly hydrated cornea.

Keywords: keratoplasty, deswelling, corneas, microkeratome

\section{Introduction}

Lamellar keratoplasty is becoming an increasingly applied surgical procedure. ${ }^{1-3}$ The reasons are that new concepts of posterior lamellar keratoplasty (ie, Descemet's stripping endothelial keratoplasty or deep posterior lamellar keratoplasty) have been introduced and developed by Melles et al, ${ }^{4,5}$ Terry and Oustey, ${ }^{6}$ and Price. ${ }^{7}$ Furthermore, modern microkeratomes have been applied for anterior lamellar keratoplasty resulting in more predictable cutting depth and surface quality. ${ }^{8-13}$ As the numbers of lamellar keratoplasty procedures are increasing, eye banks are increasingly asked to provide precut lamellar corneal tissue. With relative shortage of corneas, ${ }^{14}$ precut lamellar processing allows two lamellar grafts from one cornea. Several studies have been conducted showing the advantages and disadvantages of preserving precut donor tissue in cold storage. ${ }^{15-19}$

In Europe, the most commonly used storage method is organ culture. ${ }^{20}$ This method offers distinct advantages over cold storage such as valid assessment of the
Department of Ophthalmology, LudwigMaximilians-Universität, Mathildenstr 8, 80336 München, Germany

Tel +49 895 I60 38II

Fax +49895 I60 5I60

Email armin.wolf@med.uni-muenchen.de 
number of vital endothelial cells ${ }^{21}$ and control of sterility. ${ }^{22-24}$ Moreover, it allows long storage times of more than 30 days. ${ }^{25}$ Disadvantages of organ culture are the high costs as well as the need of a deswelling process prior to corneal transplantation. ${ }^{26,27}$ This deswelling process is necessary given that significant corneal swelling occurs during organ culture. Deswelling is usually performed by applying a hyperosmolaric medium (ie, 5\% dextran-containing medium) for an optimal time of around 32 hours. ${ }^{28}$

Similar to laser-assisted in situ keratomileusis (LASIK) and other refractive techniques, the smoothness of the interface between the donor's and the recipient's tissue is of major importance for the visual outcome of lamellar keratoplasty. ${ }^{29}$ The aim of the current study is to evaluate the lamellar dissection plane quality at different stages during the deswelling process of organ cultured donor corneas.

\section{Materials and methods}

Institutional Review Board approval was obtained in January 2010.

\section{Cornea procurement}

Eight cornea pairs (16 corneas) of seropositive donors not suitable for transplantation were obtained according to the Guidelines of the European Eye Bank Association. ${ }^{30}$ After slit lamp evaluation was conducted to ensure that the corneas were not scarred, whole eyes were thoroughly cleansed in $0.9 \% \mathrm{NaCl}$ solution, immersed in $5 \%$ polyvinylpyrrolidone iodine, and rinsed again with sodium chloride solution. Corneoscleral discs were removed, fixed on a cornea disc holder, and stored in $50 \mathrm{~mL}$ tissue culture flasks (Primaria ${ }^{\mathrm{TM}}$ Tissue Culture Flask; Falcon-Becton Dickinson Lab ware, Franklin Lakes, NJ).

\section{Organ culture storage}

Organ culture medium consisted of Eagle's minimum essential medium with Earle's salts and $25 \mathrm{mM}$ Hepes buffer (GIBCO, Glasgow, UK), containing 2\% fetal calf serum, $2 \mathrm{mM}$ glutamine, $100 \mathrm{U} / \mathrm{mL}$ penicillin, $0.1 \mathrm{mg} / \mathrm{mL}$ streptomycin, and $0.25 \mu \mathrm{g} / \mathrm{mL}$ amphotericin B. Corneas were cultured in a standard incubator at $37^{\circ} \mathrm{C}$ with the medium changed every 10 days. Deswelling was performed by placing the cornea discs in deswelling medium (Eagle's minimum essential medium with Earle's salts, and $25 \mathrm{mM}$ Hepes buffer, which contains $2 \%$ fetal calf serum, $2 \mathrm{mM}$ glutamine, $100 \mathrm{U} / \mathrm{mL}$ penicillin, $0.1 \mathrm{mg} / \mathrm{ml}$ streptomycin, $0.25 \mu \mathrm{g} / \mathrm{mL}$ amphotericin B, and 5\% dextran 500) (distribution of mol weights: $425-575 \mathrm{kDA}$ Sigma-Aldrich, Munich, Germany).

\section{Evaluation of interface quality at different deswelling periods}

After transferring the paired corneas into the deswelling medium, one was dissected after 72 hours, a duration which has been established to provide maximal deswelling. ${ }^{28}$ The corresponding corneas were cut at earlier time points during the deswelling process (ie, 0 hours and 48 hours). After corneal dissection, the specimens were placed back into the deswelling medium (Figure 1).

\section{Lamellar preparation of donor corneas}

In order to evaluate possible eye bank settings, two different types of microkeratomes were used.

\section{Manual microkeratome pass}

For the present study, a manual-guided microkeratome (Moria ONE, Antony, France) in combination with the artificial anterior chamber system (ALTK; Moria ONE) was used. A free cap lamellar dissection was performed by operating a precalibrated microkeratome head of $350 \mu \mathrm{m}$. The oscillation rate was 15000 $\mathrm{rpm}$. The pressure of the anterior chamber was set at $60 \mathrm{mmHg}$ and controlled by tonometry (Barraquer tonometer).

Two corneas were processed at 0 hours deswelling, three at 48 hours deswelling. Corneas were processed pairwise (ie, the corresponding five corneas were cut in the same manner at 72 hours).

\section{Automated microkeratome pass}

In a second setting, we used the AMADEUS ${ }^{\mathrm{TM}} 2$ microkeratome in conjunction with the MOZART ${ }^{\mathrm{TM}}$ anterior chamber system (SIS Surgical Instrument Systems, Port, Switzerland). In all donor corneas, an $8.5 \mathrm{~mm}$ suction ring with a $350 \mu \mathrm{m}$ cutting head was applied. The blade oscillation rate was 11000 rps and the advance rate was $1.5 \mathrm{~mm} / \mathrm{sec}$. The infusion bottle height was set at $200 \mathrm{~cm}$ to achieve $>60 \mathrm{mmHg}$ of pressure inside the artificial anterior chamber. The pressure was measured using a Barraquer tonometer. The "free cap" modus on the microkeratome control unit was activated. After corneal dissection, the specimen was placed back into deswelling medium (Figure 1).

Six corneas were processed using automated pass (variable swelling times: 0 hours [one] and 48 hours [two]). The corresponding corneas were processed at 72 hours.

\section{Electron microscopy}

Corneal specimens for scanning electron microscopy (SEM) were postfixed in osmium tetroxide 2\% (Dalton's fixative), dehydrated in ethanol, dried to the critical point, 


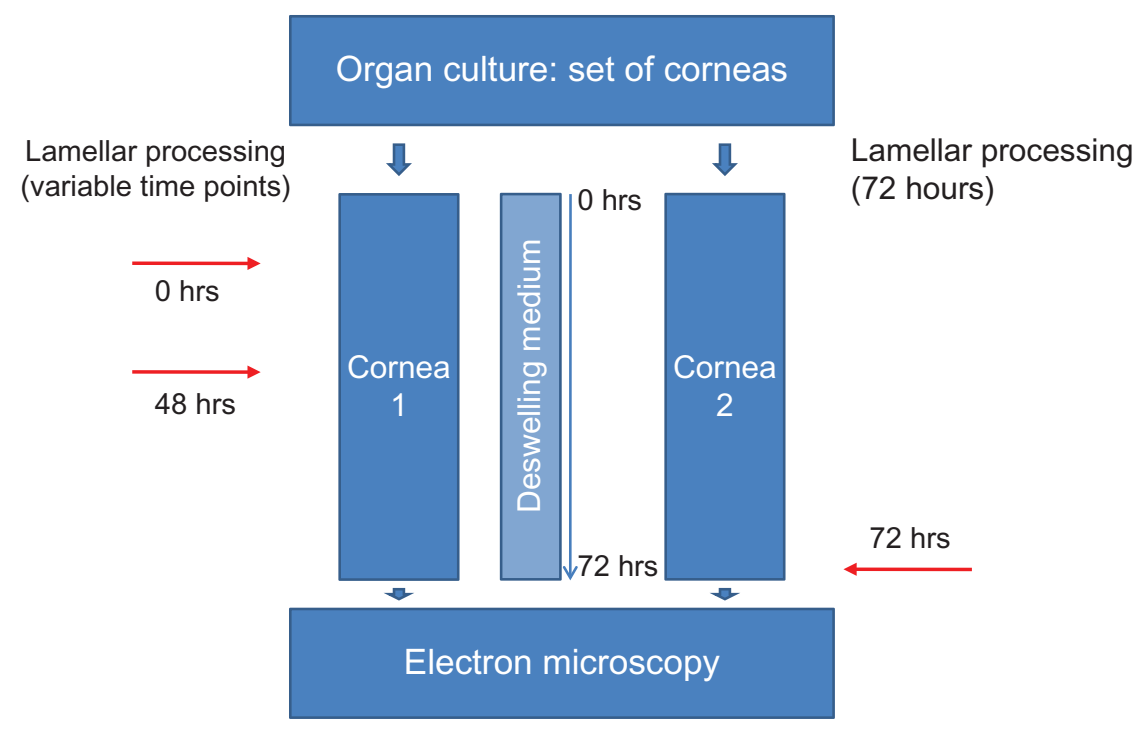

Figure I Flowchart of the study design.

Notes: Eight paired human corneas not suitable for transplantation were examined. While one cornea of the donor was cut after a variable deswelling time $(0$ hours, or 48 hours) and thereafter placed back into deswelling medium, the other cornea was processed after 72 hours of deswelling. SEM was performed after 72 hours in deswelling medium for each cornea and surface roughness of SEM images was compared thereafter by two methods.

Abbreviation: SEM, scanning electron microscopy.

sputter-coated in gold, and photographed using a ISM-35 CF electron microscope (JEOL, Tokyo, Japan).

SEM images were acquired from the central corneal bed, and additional images of the stromal bed of the cap were taken using manually guided microkeratome. Caps of mechanically cut corneas were not evaluated due to the increased likelihood of bacterial contamination in our setting.

\section{Assessment of corneal interface quality Roughness Average Score (RAS)}

The central $100 \mathrm{X}$ SEM image of the corneal interface was analyzed with the Scanning Probe Image Processor software (SPIP v.4.8.1; Image Metrology, Lyngby, Denmark) as published previously. ${ }^{10}$ In brief, this software allows for an assessment of surface smoothness by calculating the height of surfaces from the fractal dimensions. For each sample, the analysis of the surface was performed by carrying out the Roughness Average Score (RAS) function of the software.

\section{Individual surface roughness analysis (Individual scoring)}

Individual Scoring (IS) was performed as published previously. ${ }^{10}$ Images were graded by three masked observers who were asked to rate the overall smoothness or roughness of the images, while noting chatter lines and tissue tags of the stromal bed. The photographs were labeled 1-9 without disclosing the group assignment, and were presented in a random order to the masked observers. Each sample was graded as follows: $1=$ smoothest samples among all; $2=$ next smoothest; 3 = median group; $4=$ rough, but not worst; 5 = roughest samples among all. Of each sample, the mean grading from all three observers was calculated.

\section{Statistical analysis}

Statistical analysis was performed using the Statistical Package for the Social Sciences version 19.0 (SPSS, Inc., Chicago, IL). For calculation of differences in IS, a series of paired samples $t$-tests were applied because observer grading as mentioned above was based upon normal distribution. For analysis of RAS, non-parametric tests were computed.

\section{Results \\ Reproducibility of interface quality assessment}

To evaluate the reproducibility of the interface assessment, both methods (RAS and IS) were compared (see Figure 2). Spearman's rank correlation coefficient was calculated to be $r_{s}=0.66$.

\section{Overall analysis}

Overall 16 surfaces were analyzed: three obtained at 0 hours deswelling; five at 48 hours deswelling; and eight after 72 hours of deswelling. Surface roughness analysis showed a trend of smoother interface when cutting the cornea after the shortest deswelling time ( 0 hours) (IS and RAS analysis) (RAS 0 hours: 16.5 [mean], 16.1[median], standard deviation 


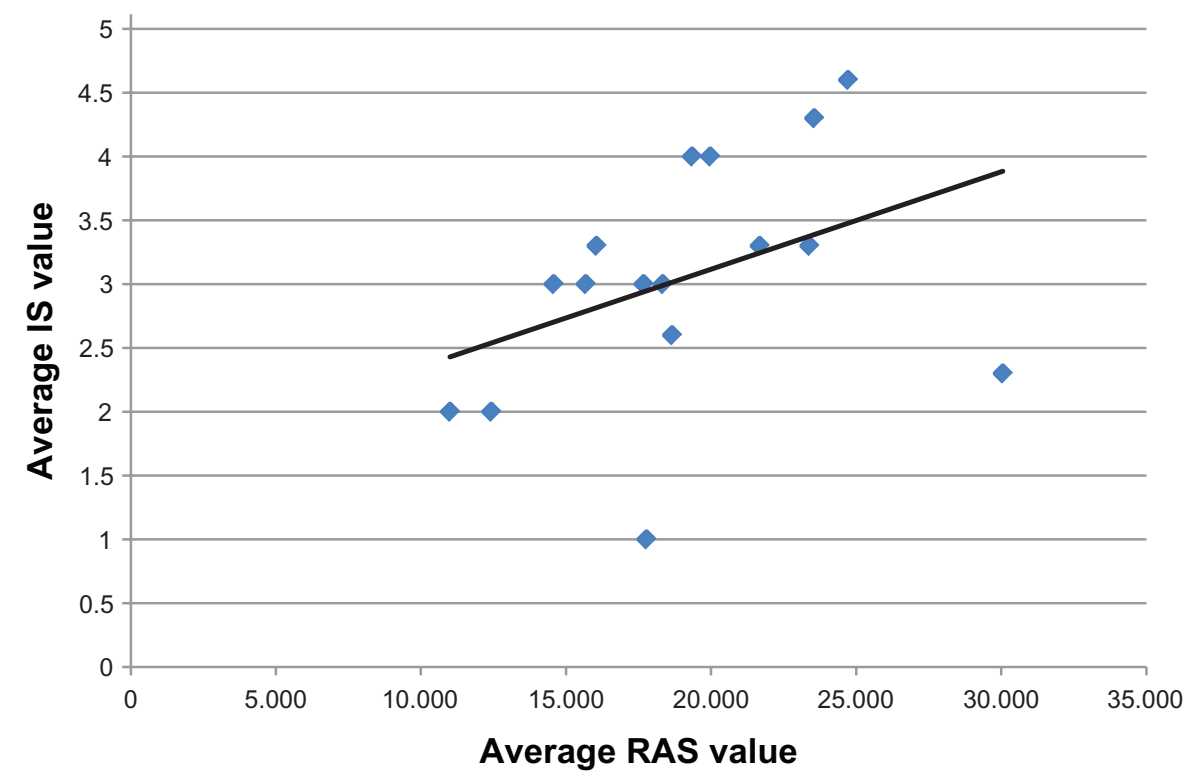

Figure 2 Comparison of the two methods used to interpret surface quality (IS by blinded graders and RAS using the SPIP software [Image Metrology, Lyngby, Denmark]). Note: There was a correlation of these two methods with Spearman's rank correlation coefficient $\left(r^{2}=0.66\right)$.

Abbreviations: SPIP, Scanning Probe Image Processor; IS, individual score; RAS, roughness average score.

1.1; RAS 48 hours: 17.4 [mean], 18.3 [median], \pm 2.9 ; RAS 72 hours: 21.0 [mean], 22.5 [median], \pm 6.0 ; IS 0 hours: 2.8 [mean], \pm 1.5 ; IS 48 hours: 2.9 [mean], \pm 0.7 , IS 72 hours: 3.4 [mean], \pm 1.0 ) (see Figure $3 \mathrm{~A}$ and B). Cutting corneas after 72 hours (longest deswelling period) resulted in the roughest surface (RAS and IS analysis); however, the difference between the groups was not statistically significant. Corneas processed after 48 hours showed a rougher surface than corneas cut before the deswelling process ( 0 hours) both in the IS and RAS analysis. A statistically significant difference was obtained only for IS between 0 hours and 48 hours ( $t$-test for paired samples, $P=0.001)$. However, the difference of surface quality between 48 and 72 hours of deswelling was only minimal (see Figure 3 ).

\section{Comparison of mechanic versus automated microkeratome pass}

All data obtained were additionally analyzed according to manual and automated dissection. There was no statistically significant difference between the two groups (Fisher's exact test).

\section{Discussion}

The trend away from full thickness penetrating keratoplasty to lamellar keratoplasty and the relative shortage of donor tissue has raised the question of whether eye banks should provide precut lamellar donor tissue. ${ }^{15,31-33}$ If this were realized, the anterior and posterior sections of a single cornea could be used for different recipients.
While several studies are available for precut donor material in cold storage, ${ }^{15,16,34,35}$ little is known about lamellar preparation in organ culture. In Europe, organ culture is the most common storage method for corneas. ${ }^{20}$ There are several advantages of organ culture including the valid assessment of cell vitality; however, a deswelling process before transplantation is needed. On the other hand, similar to LASIK, the surface quality of the transplanted corneal lamella may play an important role for the visual outcome. ${ }^{29}$

We designed this study to determine what time point of microkeratome dissection during deswelling after organ culture provides the best surface quality. There was a statistically significant smoother interface upon individual scoring for corneas processed before the deswelling process as compared to corneas processed at a later time point. Although the two methods used showed good reproducibility, we failed to detect a statistically significant advantage for software processed surface analysis. This might be due to different testing of the data for statistical reasons and due to small numbers. However, both individual scoring and computerized roughness analysis methods revealed a trend of smoother interface in earlier processed corneas with no differences between manual and automated microkeratome dissection.

One possible explanation for this finding could be the anatomy of the cornea - in the anterior stroma thin collagen lamellae run obliquely to the corneal surface. ${ }^{36,37}$ One could speculate that these lamellae are tenser in a swollen state, 


\section{A RAS-score}

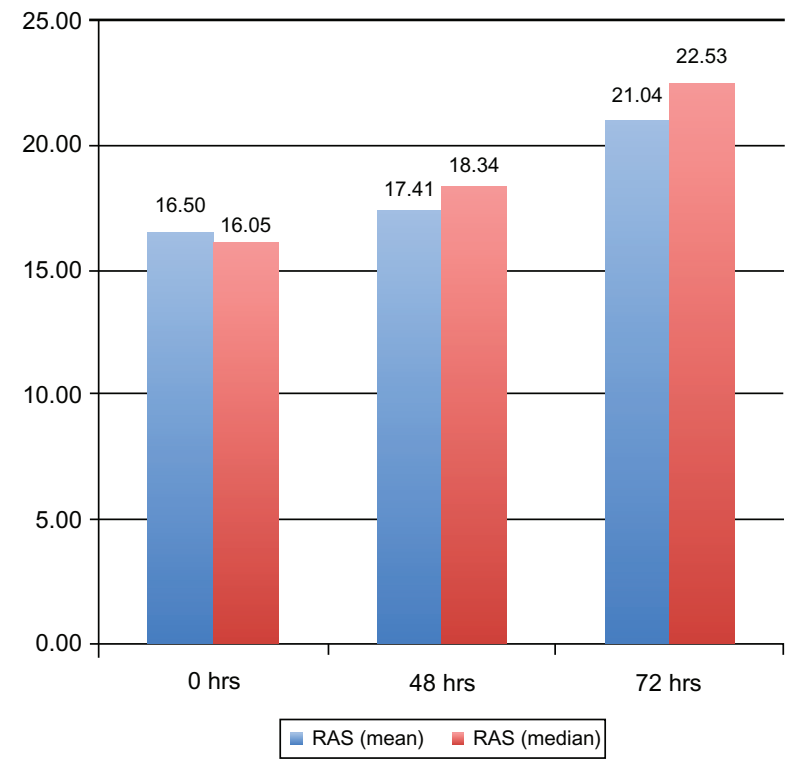

\section{B IS-score}

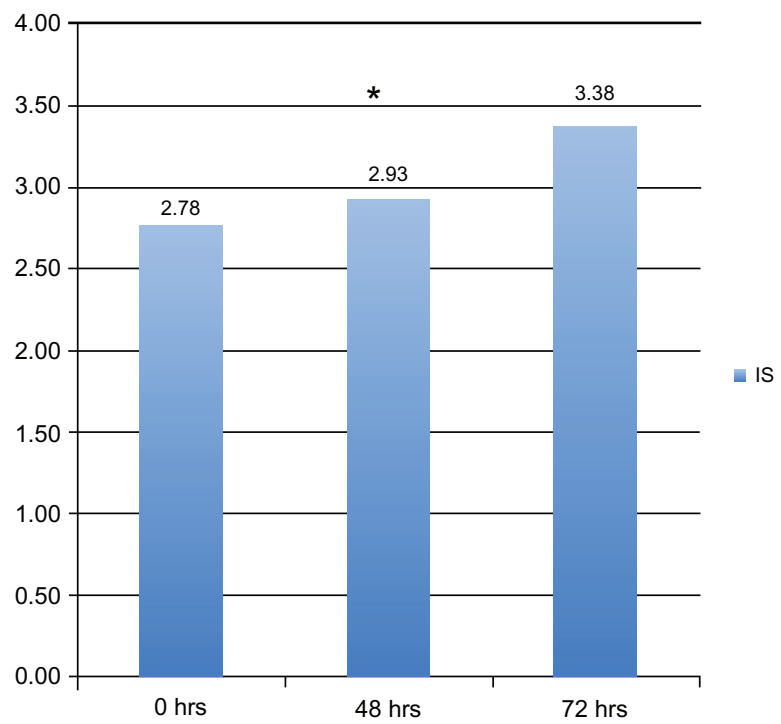

Figure 3 Analysis of surface quality of all specimens cut at 0 hours, 48 hours, and 72 hours of deswelling.

Notes: Computerized analysis (RAS): A, mean (blue), median (red) as well as the results of three blinded graders (IS): $\mathbf{B}$ revealed a trend of smoother dissection plane of corneas processed during early deswelling ( 0 hours). For difference between 0 hours and 48 hours a statistically significant $P$-value $(P<0.01)$ was calculated using paired $t$-tests.

Abbreviations: RAS, roughness average score; IS, individual scoring.

thus providing a better condition for dissection. Additionally, a more edematous state of the cornea leads to a larger space between the collagen fibrils therefore minimizing the number of damaged fibrils during the microkeratome pass. We believe this should result in a smoother interface.

Another reason could be the fact that small irregularities which occur during the microkeratome pass may diminish due to the volume reduction of the corneal stroma during the deswelling process.

We are aware of the fact that the limited count of investigated corneas is a weak point in our study. Statistical analysis did not show a significant difference for all data obtained, mainly due to the small numbers of corneal pairs used in the study. However, as we investigated human corneas under realistic eye bank conditions, there are only a limited amount of human corneas available to undertake such a study.

In conclusion, our findings support the notion that lamellar dissection of edematous corneas may result in a smoother cutting plane than dissection at the end of the deswelling process in organ cultured corneas. This finding should be taken into account when implementing the supply of precut lamellar corneal tissue into an eye bank setting while working with organ culture. Further studies are needed to ensure that our findings regarding the cutting plane quality goes along with equal accuracy of donor lamellar thickness.

\section{Disclosure}

The authors report no conflicts of interest in this work.

\section{References}

1. Tan DT, Mehta JS. Future directions in lamellar corneal transplantation. Cornea. 2007;26(9 Suppl 1):S21-S28.

2. Tan DT, Parthasarathy A. Deep anterior lamellar keratoplasty for keratoconus. Cornea. 2007;26(8):1025-1026.

3. Anshu A, Parthasarathy A, Mehta JS, Htoon HM, Tan DT. Outcomes of therapeutic deep lamellar keratoplasty and penetrating keratoplasty for advanced infectious keratitis: a comparative study. Ophthalmology. 2009;116(4):615-623.

4. Melles GR, Eggink FA, Lander F, et al. A surgical technique for posterior lamellar keratoplasty. Cornea. 1998;17(6):618-626.

5. Melles GR, de Sera JP, Eggink CA, Cruysberg JR, Binder PS. Bilateral, anterior stromal ring opacity of the cornea. Br J Ophthalmol. 1998; 82(5):522-525.

6. Terry MA, Ousley PJ. Rapid visual rehabilitation after endothelial transplants with deep lamellar endothelial keratoplasty (DLEK). Cornea. 2004;23(2):143-153.

7. Price FW Jr. Air lamellar keratoplasty. Refract Corneal Surg. 1989; 5(4):240-243.

8. Sarayba MA, Ignacio TS, Tran DB, Binder PS. A $60 \mathrm{kHz}$ IntraLase femtosecond laser creates a smoother LASIK stromal bed surface compared to a Zyoptix XP mechanical microkeratome in human donor eyes. J Refract Surg. 2007;23(4):331-337.

9. Sarayba MA, Tungsiripat T, Sweet PM, Chuck RS. A portable microkeratome for harvesting the human anterior corneal surface. Cornea. 2004;23(5):443-446.

10. Sarayba MA, Ignacio TS, Binder PS, Tran DB. Comparative study of stromal bed quality by using mechanical, IntraLase femtosecond laser 15- and 30-kHz microkeratomes. Cornea. 2007;26(4):446-451.

11. Sarayba MA, Juhasz T, Chuck RS, et al. Femtosecond laser posterior lamellar keratoplasty: a laboratory model. Cornea. 2005;24(3): 328-333.

12. Binder PS, Lambert RW, Koepnick RG, Littlefield TR. Comparison of the UniversalKeratome and the Automated Corneal Shaper. Cataract Refract Surg. 1996;22(9):1175-1188. 
13. Binder PS, Moore M, Lambert RW, Seagrist DM. Comparison of two microkeratome systems. J Refract Surg. 1997;13(2):142-153.

14. Golchet G, Carr J, Harris MG. Why don't we have enough cornea donors? A literature review and survey. Optometry. 2000;71(5):318-328.

15. Kitzmann AS, Goins KM, Reed C, Padnick-Silver L, Macsai MS, Sutphin JE. Eye bank survey of surgeons using precut donor tissue for Descemet stripping automated endothelial keratoplasty. Cornea. 2008;27(6):634-639.

16. Chen ES, Terry MA, Shamie N, Hoar KL, Friend DJ. Precut tissue in Descemet's stripping automated endothelial keratoplasty donor characteristics and early postoperative complications. Ophthalmology. 2008;115(3):497-502.

17. Terry MA, Saad HA, Shamie N, et al. Endothelial keratoplasty: the influence of insertion techniques and incision size on donor endothelial survival. Cornea. 2009;28(1):24-31.

18. Terry MA, Shamie N, Chen ES, Phillips PM, Hoar KL, Friend DJ. Precut tissue for Descemet's stripping automated endothelial keratoplasty: vision, astigmatism, and endothelial survival. Ophthalmology. 2009; 116(2):248-256.

19. Rose L, Briceno CA, Stark WJ, Gloria DG, Jun AS. Assessment of eye bank-prepared posterior lamellar corneal tissue for endothelial keratoplasty. Ophthalmology. 2008;115(2):279-286.

20. Pels LMH, Tullo A. European Eye Bank Association Directory. 2000.

21. Sperling S. Evaluation of the endothelium of human donor corneas by induced dilation of intercellular spaces and trypan blue. Graefes Arch Clin Exp Ophthalmol. 1986;22(5)4:428-434.

22. Thuret G, Carricajo A, Chiquet C, et al. Sensitivity and rapidity of blood culture bottles in the detection of cornea organ culture media contamination by bacteria and fungi. Br J Ophthalmol. 2002;86(12): $1422-1427$.

23. Thuret G, Carricajo A, Vautrin AC, et al. Efficiency of blood culture bottles for the fungal sterility testing of corneal organ culture media. Br J Ophthalmol. 2005;89(5):586-590.

24. Zanetti E, Bruni A, Mucignat G, Camposampiero D, Frigo AC, Ponzin D. Bacterial contamination of human organ-cultured corneas. Cornea. 2005;24(5):603-607.

25. Redbrake C, Salla S, Frantz A. Changes in human donor corneas preserved for longer than 4 weeks. Cornea. 1998;17(1):62-65.
26. van der Want HJ, Pels E, Schuchard Y, Olesen B, Sperling S. Electron microscopy of cultured human corneas. Osmotic hydration and the use of a dextran fraction (dextran T 500) in organ culture. Arch Ophthalmol. 1983;101(12):1920-1926.

27. Borderie VM, Baudrimont M, Lopez M, Carvajal S, Laroche L. Evaluation of the deswelling period in dextran-containing medium after corneal organ culture. Cornea. 1997;1692:215-223.

28. Wolf AH, Welge-Lüen UC, Priglinger S, et al. Optimizing the deswelling process of organ cultured corneas. Cornea. Jun 2009; 28(5):524-529.

29. Vinciguerra P, Azzolini M, Airaghi P, Radice P, De Molfetta V. Effect of decreasing surface and interface irregularities after photorefractive keratectomy and laser in situ keratomileusis on optical and functional outcomes. J Refract Surg. 1998;14(2 Supp1):S199-S203.

30. Alvarez de Toledo J AW, Ponzin D, Tulo A. EEBA Minimum Standards. European Eye Bank Association Homepage [article on the Internet]. 2007 [cited 2007]. http://www.europeaneyebanks.org/portal. Accessed. June 26, 2012.

31. Gorovoy MS. Precut tissue for descemet stripping automated endothelial keratoplasty. Cornea. 2008;27(6):632-633.

32. Price FW Jr. Precut tissue for descemet stripping automated endothelial keratoplasty. Cornea. 2008;27(6):630-631.

33. Terry MA. Precut tissue for descemet stripping automated endothelial keratoplasty: complications are from technique, not tissue. Cornea. 2008;27(6):627-629.

34. Price MO, Baig KM, Brubaker JW, Price FW Jr. Randomized, prospective comparison of precut vs surgeon-dissected grafts for descemet stripping automated endothelial keratoplasty. Am $J$ Ophthalmol. 2008;146(1):36-41.

35. Brown JS, Wang D, Li X, et al. In situ ultrahigh-resolution optical coherence tomography characterization of eye bank corneal tissue processed for lamellar keratoplasty. Cornea. 2008;27(7):802-810.

36. Komai Y, Ushiki T. The three-dimensional organization of collagen fibrils in the human cornea and sclera. Invest Ophthalmol Vis Sci. 1991;32(8):2244-2258.

37. Ojeda JL, Ventosa JA, Piedra S. The three-dimensional microanatomy of the rabbit and human cornea. A chemical and mechanical microdissection-SEM approach. J Anat. 2001;199(Pt 5): $567-576$.
Clinical Ophthalmology

\section{Publish your work in this journal}

Clinical Ophthalmology is an international, peer-reviewed journal covering all subspecialties within ophthalmology. Key topics include: Optometry; Visual science; Pharmacology and drug therapy in eye diseases; Basic Sciences; Primary and Secondary eye care; Patient Safety and Quality of Care Improvements. This journal is indexed on Submit your manuscript here: http://www.dovepress.com/clinical-ophthalmology-journal

\section{Dovepress}

PubMed Central and CAS, and is the official journal of The Society of Clinical Ophthalmology (SCO). The manuscript management system is completely online and includes a very quick and fair peer-review system, which is all easy to use. Visit http://www.dovepress.com/ testimonials.php to read real quotes from published authors. 